\title{
Laboratory Diagnosis of von Willebrand's Disease
}

\author{
Mansyur Arif *
}

\section{Abstract}

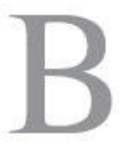

ACKGROUND: von Willebrand's disease (vWD) is an autosomally inherited bleeding disorder caused by a deficiency or abnormality of von Willebrand factor (vWF). vWF is a multimeric adhesive protein that plays an important role in primary hemostasis by promoting platelet adhesion to the subendothelium at the sites of vascular injury. It is also the carrier of factor VIII (FVIII), thus indirectly contributing to the coagulation process. Bleeding symptoms are usually mucocutaneous and postsurgical with varying severity. The diagnosis of vWD requires a personal and family history of bleeding and confirmation by laboratory analysis involving vWF antigen level, vWF ristocetin cofactor, FVIII activity, ristocetin-induced platelet aggregation, and vWF multimer analysis.

KEYWORDS: von Wilebrand's disease, von Wilebrand factor.

\section{Introduction}

von Willebrand's disease (vWD) is a hereditary abnormality of bleeding disorder that can be dominant or recessive autosomal, and is caused by a qualitative or quantitative disorder of von Willebrand factor (vWF). The revised classification of vWD identifies two major categories, characterized by quantitative (types 1 and 3 ) or qualitative (type 2) vWF defects. (1-3) vWD has a prevalence of about $1 \%$ in the general population, but the figure for clinically relevant cases is lower. (4) Also, symptoms in individuals with mild-to-moderate vWD may improve with age. In contrast, the prevalence of type $3 \mathrm{vWD}$ with the virtual absence of vWF has been estimated to be between 1 and 3 per million. (5)

vWD is characterized by mucocutaneous and postsurgical bleeding of varying severity. The disorder is very heterogenous, possibly as a result of multiple physiological functions of vWF. (5)

Two main function of $\mathrm{vWF}$ are: 1. Facilitating platelet adhesion when there is an endothelial damage by coupling the platelet membrane receptor (Glycoprotein Ib) to vascular subendothelial $(6,7) .2$. As a carrier protein for factor VIII (F VIII), an important blood coagulation protein $(4,5)$.

\section{Biosynthesis \& Structure of vWF}

The mRNA is translated as a pre-pro-polypeptide, comprises 2813 amino acids, composed of a signal peptide of 22 amino acids, a large propeptide of 741 amino acids, and a mature vWF subunit of 2050 amino acid residues with a molecular weight of $260 \mathrm{kDa}$ (Figure 1) (4,5). The mature vWF peptide consists of 2050 amino acids organized into 4 domains (A-D). Each domain contains functional residues that mediate vWF biological functions of platelet adhesion and aggregation as well as protection of FVIII from premature inactivation $(5,8)$. 


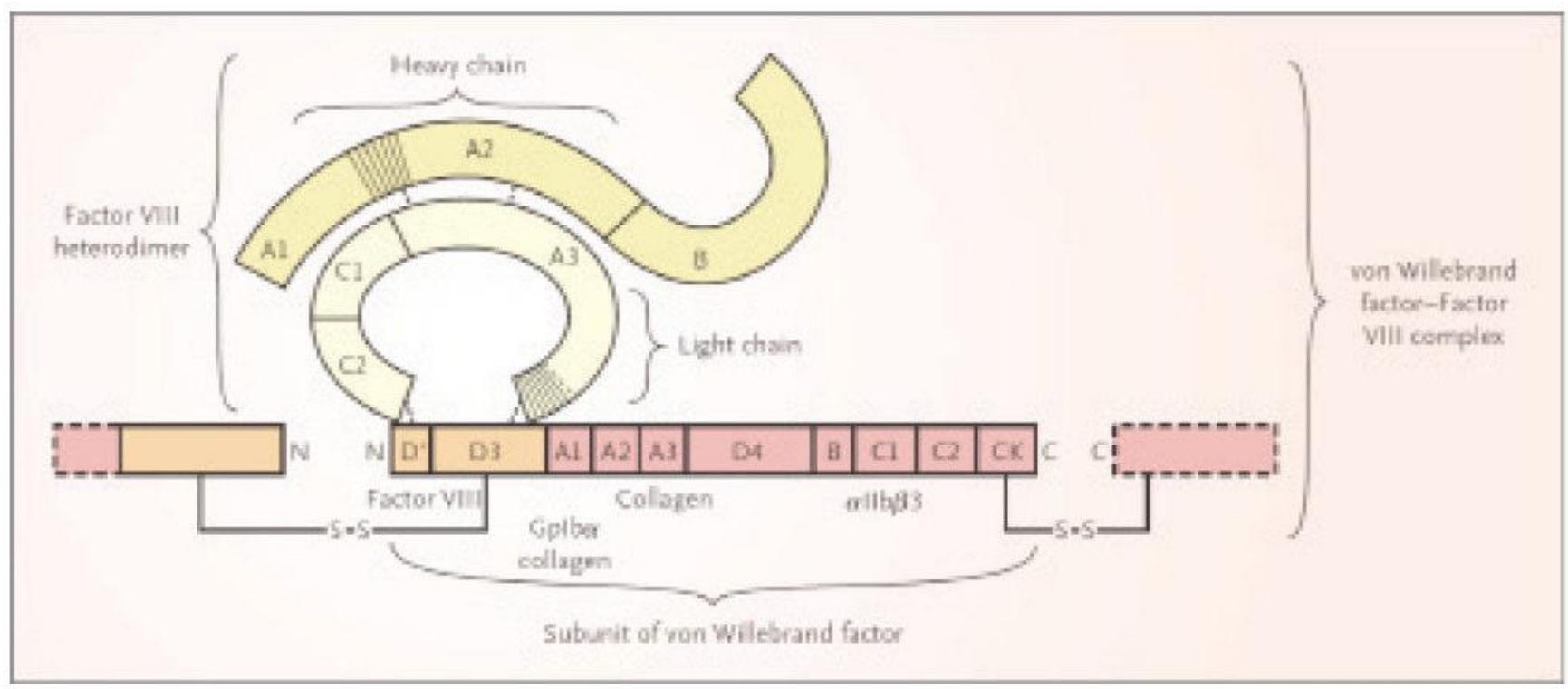

Figure 1. The structure of von Willebrand Factor (9).

Copyright (@ [2004] Massachusetts Medical Society. All rights reserved.

von Willebrand factor circulates as a complex with factor VIII, thereby protecting factor VIII from degradation by the naturally occurring anticoagulant-activated protein $\mathrm{C}$ and localizing factor VIII at the site of vascular injury (Figure 1) (9). Each mature von Willebrand factor subunit (domains D', D3, A1, A2, A3, D4, B, C1, C2, CK) dimerizes through disulfide bonds (shown as $\mathrm{S}-\mathrm{S}$ ) near the carboxy terminus (C). Each dimer further multimerizes through disulfide bonds near the amino terminus $(\mathrm{N})$. Factor VIII (domains A1, A2, B, A3, C1, C2) is cleaved before secretion and binds to von Willebrand factor as a heterodimer (9). The acidic region (cross-hatching) of the A3 domain of factor VIII and the carboxy-terminal region on the $\mathrm{C} 2$ domain bind (noncovalent bonds are shown as dotted lines) in the amino-terminal region of the von Willebrand factor subunit (domain D'-D3). The A1 and A2 domains of the heavy chain of factor VIII are noncovalently bonded to the $\mathrm{A} 3$ domain of the light chain (dotted lines). At the bottom of the figure, areas of the von Willebrand factor subunit involved in the interaction with collagen and platelet glycoproteins $\mathrm{Ib} \alpha(\mathrm{GpIb} \alpha)$ and $\alpha \mathrm{Ilb} \beta 3$ are shown (9).

vWF is synthesized at endothelial cells and megakaryocytes and it is released as a series of multimers, including ultralarge forms that are rapidly cleaved to a slightly smaller size (6). Following synthesis, the vWF is secreted by one of two distinct pathways: a persistent, constitutive release pathway or regulated release from sites of intracellular storage $(6,10)$. The majority of vWF is secreted through the constitutive pathway. vWF is stored in the platelet $\alpha$-granules, whereas in endothelial cells vWF is stored in the rod-shaped Weibel-Palade bodies (10). Only Weibel-Palade bodies or $\alpha$-granules in platelets contain fully processed and functional vWF with unusually large multimers, which are usually not found in circulation $(6,10)$.

\section{Functions of vWF}

In damaged vessels, vWF binds to the platelet $\mathrm{Gp} \mathrm{Ib}$ and to subendothelial collagen, serving as a bridge between platelets and subendothelium $(6,7)$. It also bridges between adjacent platelets in vessels with high shear stress leading to small platelet aggregates. In addition to functions in primary haemostasis, vWF also binds circulating factor VIII, independents on the size of the multimers, protecting it from proteolytic degradation in the circulation (10). In the absence of vWF, the half-life of the FVIII in the circulation decreases from 8 to 12 hours to about 2 hours. 4 vWF and factor VIII are released into the circulation, they form a tight, non-covalent complex. Each vWF monomer is able to bind one factor VIII molecule $(4,10)$. 


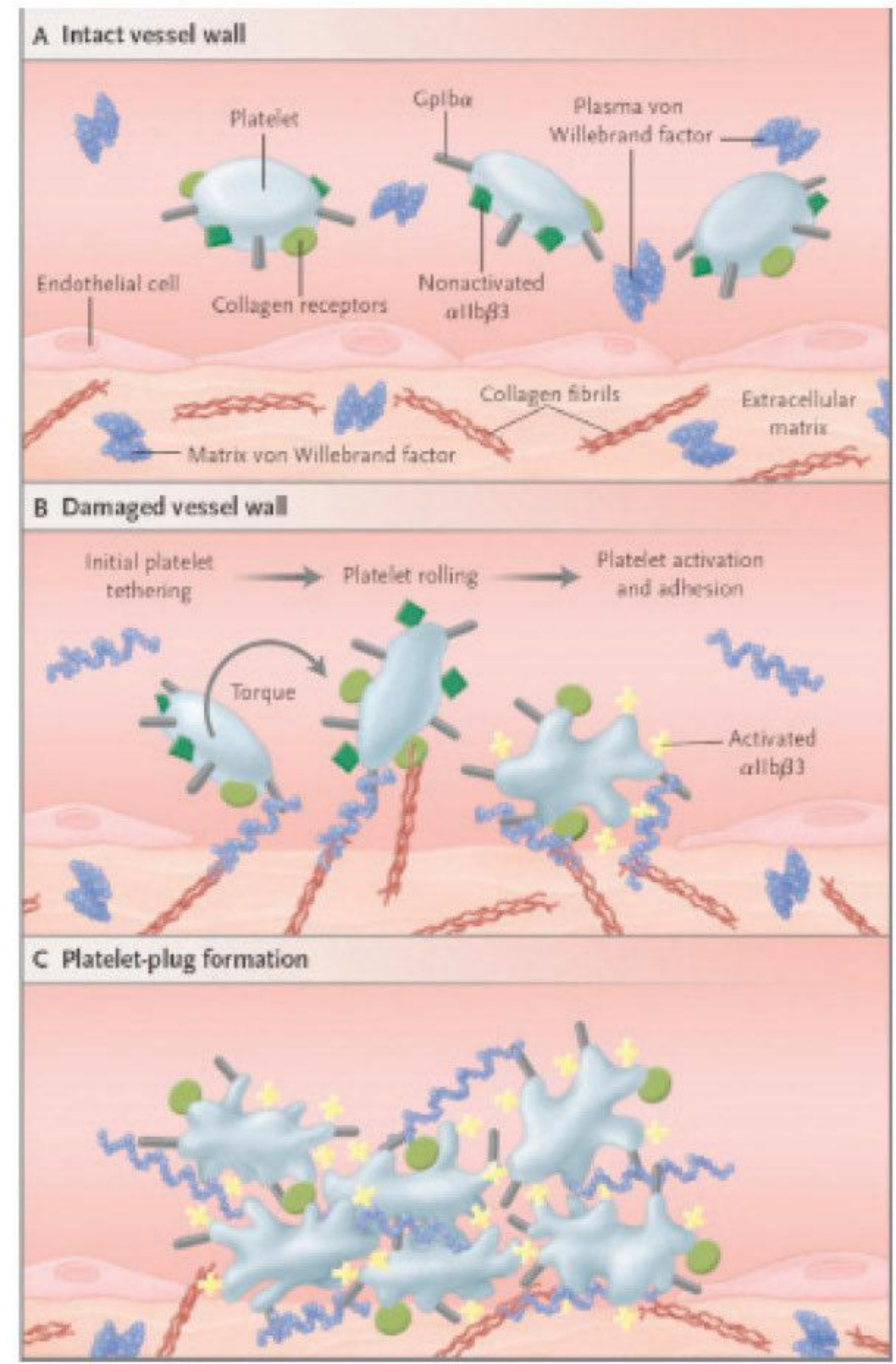

Figure 2. Simplified model of von Willebrand Factor functions in platelet-plug formation (9) Copyright (C) [2004] Massachusetts Medical Society. All rights reserved.

\section{Classification of vWD}

Classification of vWD based on the quantitative and qualitative abnormality of $\mathrm{vWF}$ is as follows:

\section{a. Quantitative Abnormality of vWF}

Type 1 and 3 is characterized by the quantitative abnormality of vWF. Type 1 is a mild disorder and the most commonly found. In this type, $40 \%$ of the members of this group carry the allele of vWD, but has a normal level of vWF. Type 3 is the most severe form and rarely occurs $(10,11)$.

\section{b. Qualitative Abnormality of vWF}

Type 2 , which consists of subtype $2 \mathrm{~A}, 2 \mathrm{~B}, 2 \mathrm{M}$, and $2 \mathrm{~N}$, involves patients with qualitative abnormality of $\mathrm{vWF}$. Type 2 includes mild to moderate abnormality, and characterized my moderate symptoms (10). 
Type $2 \mathrm{~A}$ is characterized by a decreased function of $\mathrm{vWF}$ and a deficiency in multimeric vWF. Type $2 \mathrm{~B}$ is characterized by an increased affinity of vWF to platelet $\mathrm{Gp} \mathrm{Ib}$. Type $2 \mathrm{M}$ is characterized by a decreased function of $\mathrm{vWF}$ without a deficiency in multimeric vWF. Type $2 \mathrm{~N}$ is characterized by an abnormality in vWF binding to F VIII $(10,12)$.

\section{Indication of vWF Tests}

These tests are usually applied for patients with a suspected bleeding tendency, such as mucocutaneous bleeding (ecchymosis, epistaxis, gastrointestinal haemorrhage, and menorrhage) or post surgical bleeding $(6,7)$, with normal platelet count, prolonged bleeding time (BT), normal prothrombin time (PT) whereas the activated partial thromboplastin time (aPTT) may be prolonged to a variable degree, depending on the plasma F VIII levels. (4)

\section{Laboratory Diagnosis}

A patient who presents with either spontaneous or postoperative hemmorrhage or a family history of the same should undergo a detailed evaluation of personal and family history, physical examination, and laboratory testing for a bleeding disorder (5). vWD is suspected in a patient with clinical evidence of mucocutaneous bleeding (ecchymosis, epistaxis, gastrointestinal haemorrhage, and menorrhagia) or postsurgical bleeding $(6,7)$. The large number of assays available reflects the wide spectrum of function of $\mathrm{vWF}$, and no test by itself is sensitive or specific enough to diagnose all patients. The correct classification of a patient with vWD is important not only to predict the hemorrhagic risk, but also the treatment will differ substantially on this basis. Patients with type $2 \mathrm{vWD}$ offer the greatest challenge, in terms of both diagnosis and therapeutic management. vWF and factor VIII are both acute phase reactants, increasing with stress, trauma, estrogen treatment, or pregnancy, however these factors are unlikely to obscure diagnosis in patients with more severe quantitative or qualitative defects (6). Classically screening tests, such as bleeding time (BT) and aPTT, are combined with specific tests such as vWF antigen (vWF:Ag) level, vWF activity and factor VIII activity (2).

\section{SCREENING ASSAYS}

These tests are usually applied for patients with a suspected bleeding tendency and Table 1 summarizes the different steps for diagnosing $\mathrm{vWD}(4)$.

\section{A. Bleeding Time}

This procedure is performed using a disposable apparatus to make a cut on the forearm, and the result is reported as the time taken for the cut to seal $(6,7)$ There are some disadvantages of bleeding time such as, the relatively low sensitivity and specificity of bleeding time for $\mathrm{vWD}$, its low efficacy as a predictor of surgical bleeding, operator dependence and the inconvenience to the patient. Also bleeding time may be normal in many patients with vWD $(4,7)$. Bleeding time helps to the diagnosis of vWD if it is prolonged. After replacement therapy with vWF, the bleeding time may not be corrected, but surgical bleeding is generally prevented therefore monitoring of bleeding time is not recommended $(7,13)$.

\section{B. Activated Partial Thromboplastin Time (aPTT)}

The aPTT is sensitive to deficiencies in factor VIII:C. Especially in patients with mild disease, the diagnosis of vWD may be missed by routine aPTT screening. It is important to know that a normal aPTT will not exclude $\mathrm{vWD}$. If $\mathrm{vWD}$ is genuinely suspected, specific and sensitive assays should be performed $(6,10)$.

\section{Platelet Count}

Usually platelet count is a component of a complete blood count when an automated blood counter is used. Both platelet count and platelet size should be evaluated. Patients with type $2 \mathrm{~B}$ vWD and platelet type pseudo vWD will often present with a mild thrombocytopenia $(7,13)$.

\section{Automated Platelet Function Analyzer (PFA)}

This test is used to evaluate the platelet function. In vitro techniques are developed to replace the bleeding time. The PFA-100 (Dade-Behring, Liederbach, Germany) is a new platelet function analyzer to evaluate platelet adhesion and aggregation to collagen in a whole blood assay under high shear conditions. This instrument uses citrate-anticoagulated whole blood flow through a capillary device to mimic the high shear stress conditions that occur in vivo $(10,14)$. The PFA-100 gives a single end-point reading, when the blood flow through the capillary ceases, as a result of platelet adhesion and subsequent aggregation following exposure to platelet agonists coated onto a membrane in a disposable cartridge device. This end-point is 
called the closure time. PFA closure times are sensitive to multiple factors, such as; vWF function, platelet count, platelet function, and haematocrit $(14,15)$. The PFA is very sensitive to disturbances in vWF and to the presence of vWD. Reported data suggests that the sensitivity of the PFA-100 for vWD is around $90 \%$ $(14,15,16,17)$.

\section{CONFIRMATORY LABORATORY TESTS}

\section{Factor VIII Procoagulant Activity}

The level of factor VIII:C should always be considered when evaluating patients for vWD. It is measured by the ability of patient plasma to correct the aPTT when diluted with factor VIII deficient plasma (10). An assessment of factor VIII:C will help to determine the severity of vWD, as this may suggest a low level of vWF. Factor VIII:C will also help to differentiate type $2 \mathrm{~N}$ vWD and haemophilia A. However a normal factor VIII:C level is not sufficient to exclude vWD because it may be normal in some type 1 and $2 \mathrm{vWD}$ (18).

\section{3. vWF NON-FUNCTIONAL ASSAYS}

\section{Antigen Assay (vWF:Ag)}

This is used to determine the total level of vWF protein and can be determined by immunoelectrophoresis, immunoradiometric, or more common method, such as ELISA (enzyme-linked immunosorbent assay) $(1,8)$. Average levels of vWF antigen (vWF:Ag) obtained by each laboratory vary, but usually a level of 45-50 $\mathrm{U} / \mathrm{dl}$ is the lower limit of normal with a normal range of 50-200 U/dl. The ratio of factor VIII:C to vWF:Ag also varies but generally ranges from 0.7 to 2.2 . Plasma levels of vWF:Ag is determined by carbohydrate residues attached to the protein, lower levels are noted in patients with blood type $\mathrm{O}(18)$. In order to differentiate this situation from vWD type 1, some laboratories use blood group-specific reference ranges. A low vWF:Ag level alone will provide no information about vWD subtype. Patients with type $2 \mathrm{vWD}$ may have normal vWF:Ag levels, consequently any clinical diagnosis solely on this assay is not recommended (18).

\section{4. vWF FUNCTIONAL ASSAYS}

\section{A. Ristocetin Cofactor Activity (vWF:RCo)}

The assay for ristocetin cofactor activity (vWF:RCo) quantitatively determines the ability of plasma vWF to bind to normal platelets in the presence of a compound called ristocetin and has some capacity preferentially to recognize HMW forms of vWF (18). Ristocetin, an antibiotic that induces an activation of vWF, then it binds platelets by the platelet $\mathrm{GpIb}$ receptor, similar to the in vivo interaction between subendotheial tissuebound vWF with platelets $(1,3,8)$. This test is the most sensitive and specific assay for $\mathrm{VWD}$. However the assay is a time-consuming procedure and more importantly is the problem of its reproducibility, with both high interassay and interlaboratory variability $(10,18)$. A simpler ELISA alternative of ristocetin cofactor assay has also been described. This employs a monoclonal antibody to the GpIb binding site of vWF for estimating qualitative vWF defects (18).

Recently a sensitive vWF:RCo ELISA assay using a recombinant fragment of the a-subunit of $\mathrm{GpIb}$ for the diagnosis of patients with low vWF levels was described. vWF: RCo is sensitive to the loss of both intermediate and HMW multimers. Thus, patients with type $2 \mathrm{~A}$, type $2 \mathrm{~B}$ and type $2 \mathrm{M} v W D$ will have lower vWF:RCo than vWF:Ag test results $(<0.6)$ because of the relative absence of high and intermediate vWF molecular weight forms (type $2 \mathrm{~A}$ ), relative absence of HMW vWF forms (type2B) or functionally defective vWF forms (type $2 \mathrm{M})(10,12,18)$. Ristocetin cofactor activity is also generally used to follow a patient's response to therapy (18).

\section{B. Ristocetin-induced Platelet Aggregation (RIPA)}

This assay is a qualitative test that evaluates the rate or extent of agglutination of patient's platelet-rich plasma for sensitivity to ristocetin at various concentrations. At least two or three concentrations of ristocetin are used over a range between 0.5 to $1.5 \mathrm{mg} / \mathrm{ml}$ (10). RIPA is dependent on both the level and the function of vWF and platelets present in the test plasma, as well as on the amount of ristocetin. Normal individuals will show platelet aggregation at or above $1.0 \mathrm{mg} / \mathrm{ml}$ ristocetin but typically not below this concentration $(18,20)$.

The level of platelet aggregation in type $1 \mathrm{vWD}$ will depends on the level of vWF. Although RIPA test is somewhat insensitive to mild quantitative deficiencies, patients with severe type $1 \mathrm{vWD}(<15 \% \mathrm{vWF})$ will tend to show no aggregation with $1.0 \mathrm{mg} / \mathrm{ml}$ or below ristocetin and no or mild aggregation at $1.5 \mathrm{mg} / \mathrm{ml}$. Patients with severe type $2 \mathrm{~A}$ vWD will show a similar pattern of no aggregation with up to $1.0 \mathrm{mg} / \mathrm{ml}$ ristocetin and no or little aggregation at $1.5 \mathrm{mg} / \mathrm{ml}(10,18,20)$. In contrast variants of vWD with 'gain of function' mutations (type $2 \mathrm{~B}$ and platelet-type pseudo- vWD) show an enhanced aggregation response, typically aggregating with $0.6 \mathrm{mg} / \mathrm{ml}$ or below of ristocetin. RIPA is used primarily to assess if there is a gain of function mutation in the patient with $\mathrm{vWD}$. Low dose ristocetin 
aggregation cannot differentiate type $2 \mathrm{~B}$ vWD from platelet-type pseudo vWD, and mixing studies using patient plasma and donor platelets have been devised $(18,20)$. Patients with type 3 vWD will not show platelet aggregation at any ristocetin concentration as well as in patients with Bernard-Soulier syndrome in which platelets are missing the $\mathrm{GpIb}$ receptor (18).

\section{C. von Willebrand Collagen-binding Activity} (vWF: CB)

vWF contains collagen-binding sites, and collagenbinding activity (vWF:CB) is an important function of $\mathrm{vWF}$ to tether to platelets at sites of vascular injury (10). This quantitative and qualitative assay is performed by an ELISA procedure, in which type I or type III collagen is plated into microtiter wells, and the amount of vWF captured is assayed. The vWF:CB estimates the level of vWF, but its greatest strength is its ability to detect $\mathrm{vWF}$ of primarily HMW forms which are the most functional and adhesive forms $(18,19)$. Thus, the vWF:CB contributes to the diagnosis of $\mathrm{vWD}$ by providing information on the quality of vWF present. Type $2 \mathrm{~A}$ and type $2 \mathrm{~B} \mathrm{vWD}$ will yield very low vWF:CB values because of the absence of HMW vWF forms $(19,20)$.

\section{Factor VIII Binding Assay (vWF: FVIIIB)}

FVIII binding by vWF is performed as an ELISA procedure and also involves a chromogenic assay step.

It is useful to diagnose type $2 \mathrm{~N}$ vWD and assesses the ability of vWF to bind FVIII. 1 In this ELISA format assay, ELISA platelet wells are coated with antibody to $\mathrm{vWF}$ to capture vWF from patient plasma. After washing patient-derived FVIII from the well, the ability of patient-derived $\mathrm{vWF}$ to capture recombinant FVIII (r-FVIII) is assessed using a chromogenic assay for FVIII, and the quantity of patient-derived vWF:Ag in the well is quantified by standard ELISA technique $(10,18)$. The calculated ratio of r-FVIII to vWF:Ag is expected to be in the normal range in patients with haemophilia $\mathrm{A}$, whereas it is abnormally low (ratio of less than 0.6 ) in type $2 \mathrm{~N}$ vWD (18). Alternatively, a normal level of bound vWF and FVIII with similar proportional values, giving a ratio of around 1 is consistent with a normal sample or may reflect type $2 \mathrm{~B}$ or $2 \mathrm{M} v W D$; $a$ low level of bound $\mathrm{vWF}$ and FVIII though with proportionally similar values, giving a ratio of around 1 is consistent with a type $1,2 \mathrm{~A}$ or $2 \mathrm{~B}$ $\mathrm{vWD}$, and a relative absence of both bound $\mathrm{vWF}$ and FVIII will suggest type 3 or very severe type $1 \mathrm{vWD}$ $(12,20)$.

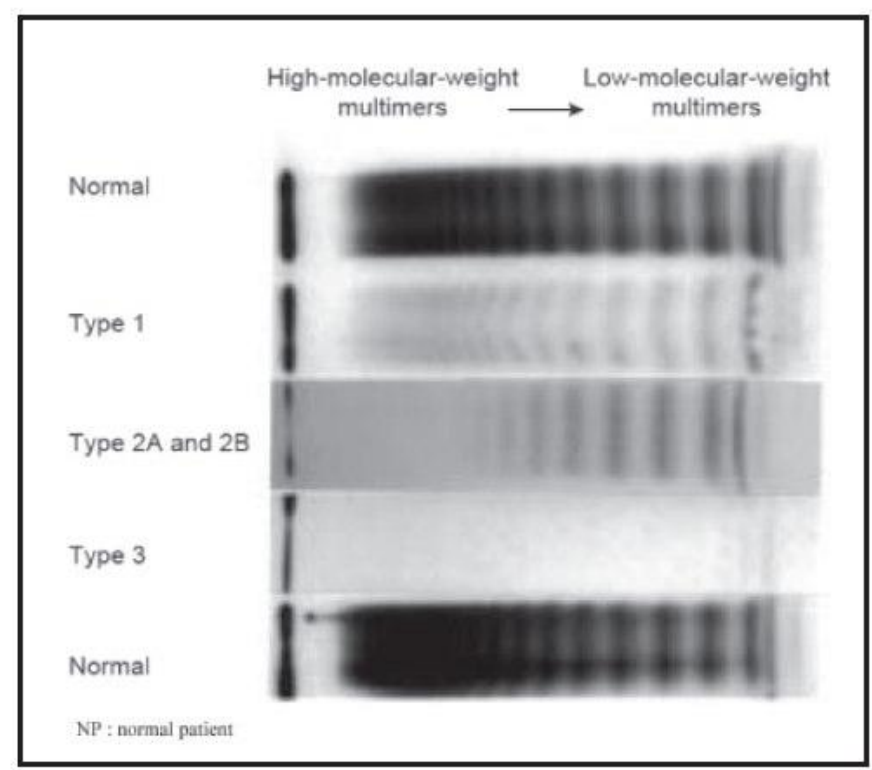

Figure 3. von Willebrand factor multimers (From Pruthi RK. A practical approach to genetic testing for von Willebrand's disease. Mayo Clin Proc. 2006;B1(5): 679-691. Used with permission.

\section{E. Multimeric Analysis of vWF}

This assay detects vWF of differing molecular weights, as well as identifying certain vWF structural abnormalities. It is performed by only a small number of expert coagulation reference laboratories, because of its complexity and cost (1). The procedure involves the agarose gel electrophoresis, and vWF multimers are imaged by immunologic methods such as autoradiography or immunoperoxidase technique (Figure 3) $(6,10)$.

Normal vWF is composed of a complex series of multimers with molecular weight ranging from 800 to $20,000 \mathrm{kDa}$, which can be analyzed by agarose gel electrophoresis. Low-resolution agarose gels distinguish vWF multimers, which are conventionally indicated as high, intermediate and low molecular weight. In types 1, $2 \mathrm{M}$ and $2 \mathrm{~N} v W D$ all multimers are present, whereas in types $2 \mathrm{~A}$ and $2 \mathrm{~B}$ the high and intermediate multimers are missing (4).

It is not appropriate to order vWF multimer assay during the initial vWD investigation process. The procedure is however, recommended when it is necessary to confirm or subtype type $2 \mathrm{vWD}$ that has been diagnosed using clinical criteria and specific quantitative and qualitative diagnostic tests. This method confirms the loss of intermediate-or HMW multimers of vWF as is characteristic of the type $2 \mathrm{~A}$ and type $2 \mathrm{~B}$ defects $(6,20)$. However, distinction between type $2 \mathrm{~A}$ and type $2 \mathrm{~B} v W D$ is not always possible using multimer analysis alone, necessitating further testing. Results of multimeric analysis may be helpful in predicting response to DDAVP treatment $(9,20)$. 
Table 1. Common laboratory findings associated with various types of vWD (8).

\begin{tabular}{|c|c|c|c|c|c|c|}
\hline & Type 1 & Type 3 & Type 2A & Type 2B & Type 2M & Type 2N \\
\hline vWF:Ag & $\downarrow \quad$ or $\downarrow \downarrow$ & $\begin{array}{c}\text { absent } \\
(<0.05 \mathrm{U} / \mathrm{mL})\end{array}$ & $\downarrow$ & $\downarrow$ & $\downarrow$ & normal or $\downarrow$ \\
\hline vWF:RCo & $\downarrow \quad$ or $\downarrow \downarrow$ & $\begin{array}{c}\text { absent } \\
(<0.05 \mathrm{U} / \mathrm{mL})\end{array}$ & $\downarrow \downarrow \quad$ or $\downarrow \downarrow \downarrow$ & $\downarrow \downarrow$ & $\downarrow \downarrow$ & normal or $\downarrow$ \\
\hline FVIII:C & normal or $\downarrow$ & $0.01-0.10 \mathrm{U} / \mathrm{mL}$ & normal or $\downarrow$ & normal or $\downarrow$ & normal or $\downarrow$ & $\downarrow \downarrow \quad$ or $\downarrow \downarrow \downarrow$ \\
\hline $\begin{array}{l}\text { vWF:RCo I } \\
\text { vWF:Ag ratio }\end{array}$ & $>0.6$ & not useful & $<0.6$ & $<0.6$ & $<0.6$ & $>0.6$ \\
\hline Multimers & normal & absent & $\begin{array}{l}\text { loss of high } \\
\text { (and possibly } \\
\text { intermediate) } \\
\text { molecular weight } \\
\text { multilmers }\end{array}$ & $\begin{array}{l}\text { loss of high } \\
\text { molecular } \\
\text { weight }\end{array}$ & normal & normal \\
\hline
\end{tabular}

Table 2. Clinical and laboratory parameters used for vWD diagnosis (4).

\begin{tabular}{|l|}
\hline Patients at risk of vWD \\
\hline $\begin{array}{l}\text { Clinical history: lifelong mucocutaneous and postoperative bleeding. } \\
\text { Symptoms are sometimes present in other family members. } \\
\text { Screening tests: prolonged bleeding time (maybe normal); normal platelet count; } \\
\text { prolonged PTT (maybe normal). }\end{array}$ \\
\hline Diagnosis and definition of vWD \\
vWF antigen \\
vWF: Ristocetin cofactor activity \\
Factor VIII \\
vWF multimeric structure on low resolution gels \\
\hline Diagnosis of vWD subtype \\
\hline $\begin{array}{l}\text { Ristocetin-induced platelet agglutination (RIPA) } \\
\text { vWF multimeric structure on high resolution gels } \\
\text { Platelet vWF content } \\
\text { Factor VIII binding assay }\end{array}$ \\
\hline
\end{tabular}




\section{Summary}

von Willebrand's disease (vWD) is the most common inherited bleeding disorder, caused by mutations in the von Willebrand factor (vWF) gene resulting in deficiency or abnormality of $\mathrm{vWF}$. vWF is a multimeric adhesive protein which plays an important role in primary hemostasis by promoting platelet adhesion to the subendothelium at sites of vascular injury and it is also the carrier of factor VIII (FVIII), thus indirectly contributing to the coagulation process. The revised classification of $\mathrm{vWD}$ identifies two major categories, characterized by quantitative (types 1 and 3 ) or qualitative (type 2) vWF defects. Bleeding symptoms are usually mucocutaneous and postsurgical with varying severity. The diagnosis of $\mathrm{vWD}$ requires several laboratory tests involve vWF antigen level, vWF ristocetin cofactor, FVIII activity, ristocetin-induced platelet aggregation, and vWF multimer analysis.

\section{References:}

1. Biner B. Von Willebrand factor and von Willebrand disease. Haema 2005; 8: 405-18.

2. Piñol M, Sales M, Costa M, Tosetto $A$, Canciani MT, Federici AB. Evaluation of a new turbidimetric assay for von Willebrand factor activity useful in the general screening of von Willebrand disease. Haematologica 2007; 92: 712-3.

3. Federici AB, Canciani MT, Forza I, et al. A sensitive ristocetin co-factor activity assays with recombinant glycoprotein Iba for the diagnosis of patients with low of von Willebrand factor levels. Haematologica 2004; 89: 77-85.

4. Castaman G, Federici AB, Rodeghiero F, Mannucci PM. Von Willebrand's disease in the year 2003: towards the complete identification of gene defects for correct diagnosis and treatment. Haematologica 2003; 88: 94 108.

5. Pruthi RK. A practical approach to genetic testing for von Willebrand disease. Mayo Clin Proc 2006; 81: 679-91.

6. Lillicrap D, James P. Von Willebrand's disease: an introduction for the primary care physician. Haemophilia 2009; 47 : 1-7.
7. Hayward CP. Diagnosis and management of mild bleeding disorders. Hematology 2005; 1: 423-8.

8. Kasper CK. Von Willebrand disease. 2002 [cited 2009 Mar 25]. Available from : www.carolkasper.com/11_5_05/NWD.pdf

9. Mannucci PM. Treatment of von Willebrand's disease. N Engl J Med 2004; 351: 683-94.

10. Sadler JE, Budde U, Eikenboom JCJ, et al. Update on the pathophysiology and classification of von Willebrand disease: a report of the subcommittee of von Willebrand factor. J Thromb Haemost 2006; 4: 2103-14.

11. Sadler JE. Von Willebrand disease type $\mathrm{I}$ : a diagnosis in search of a disease. Blood 2003; 101: 2089-93.

12. Ruggeri ZM. Type IIB von Willebrand disease: a paradox explains how von Willebrand factor works. J Thromb Haemost 2003; 2: 2-6.

13. Wallach J. Interpretation of diagnostic tests. 7th ed. Philadelphia: Lippincott Williams\&Wilkins, 2000; p. 484-7.

14. Franchini M, Gandini G, Manzato F, Lippi G. Evaluation of the PFA-100 system for monitoring desmopressin therapy in patients with type 1 von Willebrand's disease. Haematologica 2002; 87: 670 .

15. Davies JR, Fernando R, Hallworth SP. Hemostatic function in healthy pregnant and preeclamptic women: an assessment using the platelet function analyzer (PFA-100®) and Thromboelastograph ${ }^{8}$. Anesth Analg 2007; 104: 416-20.

16. Koscielni J, Ziemer $\mathrm{S}$, Radtke $\mathrm{H}$, et al. A practical concept for preoperative identification of patients with impaired primary hemostasis. Clin Appl Thromb/Hemost 2004; 10 : 195-204.

17. Hayward CP, Harrison P, Cattaneo, Ortel TL, Rao AK. Platelet function analyzer (PFA)-100® closure time in the evaluation of platelet disorders and platelet function. $\mathrm{J}$ Thromb Haemost 2006; 4: 312-9.

18. Lippi G, Franchini M, Salvagno GL, Montagnana M, Pati G, Guidi GC. Correlation between von Willebrand factor antigen, von Willebrand factor ristocetin cofactor activity and factor VIII activity in plasma. J Thromb Thrombolysis 2008; 26: 150-3.

19. Popov J, Zhukov O, Ruden S, Zeschmann T, Sferruzza A, Sahud M. Performance and clinical utility of a commercial von Willebrand factor collagen binding assay for laboratory diagnosis of von Willebrand disease. Clin Chem 2006; 52): 1965-7.

20. Giannini S, Mezzasoma AM, Leone M, Gresele P. Laboratory diagnosis and monitoring of desmopressin treatment of von Willebrand's disease by flow cytometry. Haematologica 2007; 92: 1647-54.s 